\title{
Assessment of genetic resources of representatives of the genus Prunus $L$. in the conditions of the Crimea
}

\author{
Valentina Gorina ${ }^{1 *}$, Vadim Korzin ${ }^{1}$, Nikita Saplev ${ }^{1}$, and Ekaterina Melkozerova ${ }^{1}$ \\ ${ }^{1}$ Federal State Funded Institution of Sciense «The labour red banner order Nikitsky botanical gardens \\ - national scientific center of the ras», u.s. Nikita, Yalta, 298648, Russia,
}

\begin{abstract}
The aim of the work is a comprehensive assessment of the genetic resources of apricot in the Nikitsky Botanical Garden, determination of the main directions of breeding and the selection of appreciable genotypes for further most effective breeding use for creating competitive varieties, and their introduction into production. Based on the conducted studies, it was revealed that the main directions of apricot breeding at the modern level is to determine the possibility of effective targeted selection of parental pairs for creating varieties with high commercial qualities of fruits, high-yielding, resistant to abiotic and biotic stress factors, adapted to intensive cultivation technologies. It has been determined that the Zard variety, up to the second generation, transmits to its offspring late flowering and small fruiting size. The most promising apricot genotypes for cultivation in areas with unstable weather conditions in Southern Russia have been identified: Boyarin, Yaltinec, 84-951, 89-653, 99-396, 8316, 10841, 84-941, they are characterized by large or medium-sized fruits, good taste (tasting score 4.14.7 points, on a 5-point scale) and attractive appearance, late or mid-late flowering of plants. For breeding for late flowering is of interest: Zapozdalyj, Ozornik, 84-383, 84-895, 8319, 84-875; weak susceptibility to fungal pathogens: clasterosporium and moniliosis - 84-919, 84-880, 84-383, 84-915, 84-875, 84-909, 84-803, 84-769, 84-803, 89-545, 10841; drought resistance: Krokus, Yaltinec, 87-2, 84-651, 84-942, 84-988, 84-639, 89-359, 89-526, 89-166 и 89-169. Against the background of dry growing seasons, the yield of the selected breeding forms was at the level of the control variety and higher (2.6-3.9 points).
\end{abstract}

\section{Introduction}

Modern horticulture in the Russian Federation is based on economically and environmentally effictive technologies for the production of fruit products. In a market system, especially recently, priority is given to its competitiveness $[1,2]$.

The development of resource-saving technologies in horticulture will increase the efficiency of agricultural activities: the formation of crowns, harvesting, soil maintenance under plantings, their water balance, protection of plants from negative environmental

\footnotetext{
*Corresponding author: valgorina@yandex.ru
} 
factors, which requires improving the varietal composition of fruit crops. At present, in horticultural agrocenoses mainly cultivated varieties, which biological characteristics do not always correspond to the conditions of their growth. To improve the state of the horticultural industry, it is necessary to introduce varieties that are most adapted to growing conditions, more resistant to diseases and pests, and are distinguished by quality products [3, 4].

Apricot (Armeniaca vulgaris Lam.) belongs to the group of fruit crops that enter largely in the structure of world and European fruit production. More than $72 \%$ of the countries among the states that growing the widest range of stone fruit crops, such as peach, nectarine, sweet cherry, cherry, plum, cherry plum, cultivate apricot. In the world, the apricot plantations make up $9.5 \%$ of all cultivated stone fruit crops, and the gross yield of apricot fruits is $8.2 \%$. Great importance is attached to this culture in France. It is part of the French national project (ILLIAD) for food security [5]. The Russian Federation also pays great attention to the development of intensive horticulture. Stone fruit plantations are cultivated in many regions of the country and amount to 119.4 thousand hectares, moreover, $10.1 \%$ of the area is devoted to apricot, which ranks fourth among the seven stone fruit crops cultivated in Russia. In terms of gross yield (65, 8 tons), it ranks third and is equal to $12.1 \%$ [6]. Due to the increased demand for the fruits of this crop, its varieties must meet certain requirements.

To create competitive apricot varieties, it is necessary to optimize approaches to the breeding process, and at the same time it is important to study genetic resources, the diversity of which will allow the selection of the most effective initial pairs. Therefore, defining the direction of development of breeding research of apricot culture, to increase their efficiency, isolation of genotypes with high adaptive properties, regular yield and high-quality fruit products with high donor abilities is very important [7-11].

The aim of this work is a comprehensive assessment of the genetic resources of apricot in the Nikitsky Botanical Garden, determination of the main directions of breeding and the selection of promising genotypes for the further most effective breeding use during creating competitive varieties, and their manufacturing application

\section{Materials and methods}

The research was carried out during 2015-2019. on the basis of the collection plantations of the Nikitsky Botanical Garden on the Southern coast of Crimea. The object of the study was 65 genotypes of common apricot (A. vulgaris Lam.), planted in 2011. Rootstock - apricot seedlings, planting pattern $5 \times 3 \mathrm{~m}$. A widespread zoned variety Krymskiy Amur was used as a control. In research on variety study, the study of field resistance of varieties and hybrid forms of apricot to Moniliacinerea Bon. and Clasterosporium carpophilum (Lev.) Aderh.) were guided by well-known techniques.

Statistical analysis was presented as the mean value with the standard mean square error. All statistical analyzes were performed using the Microsoft Office Excel computer program.

\section{Results and discussion}

One of the main disadvantages of apricot is the short dormant period of the generative buds of most cultivars grown in production. Fluctuations in air temperature in winter-spring time cause early vegetation in plants, which contributes to the death of generative elements from frosts. All of this leads to irregular fruiting and low profitability of apricot plantations. Consequently, this culture, despite the value of the fruit, could not compete with economically more profitable breeds such as peach, sweet cherry, plum. Therefore, the main task of the breeders was to create varieties of apricot with a later flowering. Late-flowering plants are characterized by slow development; during this period their generative sphere is 
more resistant to the negative effects of frost $[12,13,14]$. In the Nikitsky Botanical Garden, have been working on the issues of breeding for late apricot flowering from the earliest time K.F. Kostina, A.M. Sholokhov, V.K. Smykov, G.A. Gorshkova, N.G. Ageeva. During the formation and comprehensive study of the apricot collection, it was revealed that the Central Asian genotypes are distinguished by the greatest diversity of this trait. A very late flowering cultivar such as Zard was selected among the introduced species. It is characterized by small, light-colored fruits with a rather large stone; in the conditions of the Crimea it stands out for its advanced resistance to drought and frost. K.F. Kostina included this variety in hybridization with large-fruited varieties that are distinguished by fruits of good taste and average flowering times. Among these varieties was the local Armenian variety Erevani (Shalakh), whose plants had high-quality, large fruits with creamy blush; and central Asian varieties: Komsomolec with large fruits with orange blush and Arzami with yellow-creamy medium-sized fruits.

Later, descendants were obtained from crossing the Zard variety with the Vynoslivyj (medium-sized orange fruits) and Yupiter (light yellow large fruits) varieties. As a result of the study of the obtained hybrid forms, promising genotypes were identified, which are characterized by medium, medium-late or late flowering of plants. Variety Zard clearly conveys to its offspring late flowering and small fruit size. The selected genotypes were recrossed with varieties, characterized by large to medium-sized fruits of good taste with bright orange, light orange skin and flesh (Naslazhdenie, Olimp, Professor Smykov) or with varieties, the fruits of which are distinguished by large creamy fruits with dense solid pulp, harmonious taste (Forum, Kostinskij) to improve the commercial qualities of the fruits

As a result, hybrids that combine medium or late flowering of plants with larger fruits were obtained. Among them, half of the hybrid forms (50\%) have a rather light color of the skin and pulp, and half are characterized by orange fruits.

A comprehensive study of hybrid forms created with the participation of the Zard variety was carried out. Weather conditions during the study period were favorable for the growth, development and fruiting of apricot plants. only exception was 2015 (Table 1.).

Table 1. Average monthly air temperature ${ }^{\circ} \mathrm{C}(2015-2019)$

\begin{tabular}{|l|l|l|l|c|c|c|c|c|c|c|c|c|}
\hline \multirow{2}{*}{ Year } & \multicolumn{10}{|c|}{ Month } \\
\cline { 2 - 13 } & I & II & III & IV & V & VI & VII & VIII & IX & X & XI & XII \\
\hline 2015 & 5.0 & 4.6 & 6.6 & 9.3 & 16.0 & 21.3 & 24.0 & 25.5 & 22.4 & 13.1 & 10.2 & 6.0 \\
\hline 2016 & 3.0 & 6.4 & 7.6 & 12.4 & 15.8 & 22.6 & 24.8 & 25.8 & 19.9 & 8.6 & 8.2 & 2.6 \\
\hline 2017 & 2.2 & 3.6 & 8.6 & 9.8 & 15.6 & 20.9 & 23.9 & 23.6 & 21.0 & 13.5 & 9.0 & 8.5 \\
\hline 2018 & 4.6 & 4.9 & 6.9 & 14.5 & 18.8 & 22.8 & 24.6 & 26.6 & 20.4 & 16.1 & 8.3 & 5.6 \\
\hline 2019 & 4.9 & 5.4 & 6.9 & 11.2 & 17.2 & 24.8 & 23.2 & 24.9 & 19.9 & 15.3 & 11.4 & 7.9 \\
\hline $\begin{array}{l}\text { Climate } \\
\text { normals }\end{array}$ & 3.1 & 3.3 & 5.3 & 10.5 & 15.4 & 19.9 & 22.8 & 22.6 & 18.4 & 13.0 & 8.9 & 5.5 \\
\hline
\end{tabular}

In the 2015, significant changes in air temperature were observed during the flowering period. The average daily air temperature did not exceed $8{ }^{\circ} \mathrm{C}$, the minimum was $2.1^{\circ} \mathrm{C}$, and the maximum was $11.5^{\circ} \mathrm{C}$ [15]. In the first generation of hybrid forms created with the participation of the Zard variety, out of six genotypes three (Arzami Sladkiy, 87-2 and Zapozdaliy) with medium-late and late flowering were distinguished. In the second generation, $65.5 \%$ of hybrid forms were selected with medium-late and late flowering periods. The latest flowering are hybrid forms 84-875, 84-919, 8319, 84-383, 84-915 and 99396. Large fruits $(67.0 \mathrm{~g})$ in the first generation stood out for the Red Crimea variety, in the second generation (50.0-73.2 g) - 15 varieties and hybrid forms. Large-fruited (60.0-73.2 g) were varieties Crocus, Yaltinets and hybrid form 84-951. In the third generation, all three 
genotypes have large fruits. The characteristics of promising hybrid forms are presented in table 2.

Table 2. Characteristics of new hybrids of apricot (2015-2019)

\begin{tabular}{|c|c|c|c|c|c|c|}
\hline № & $\begin{array}{l}\text { Variety, hybrid } \\
\text { form }\end{array}$ & $\begin{array}{c}\text { The } \\
\text { beginning } \\
\text { of } \\
\text { flowering }\end{array}$ & $\begin{array}{l}\text { The } \\
\text { beginning } \\
\text { of fruit } \\
\text { ripening }\end{array}$ & $\begin{array}{l}\text { Appearance } \\
\text { (skin color, } \\
\text { blush) }\end{array}$ & $\begin{array}{c}\text { Fruit size, } \\
\text { g. }\end{array}$ & $\begin{array}{l}\text { Fruit } \\
\text { taste, } \\
\text { score }\end{array}$ \\
\hline 1 & 2 & 3 & 4 & 5 & 6 & 7 \\
\hline 1 & Krymskij Amur (k) & $22 / 03 \pm 4$ & $11 / 07 \pm 9$ & $\begin{array}{l}\text { yel.-or., red. } \\
\text { bl, up to } 25 \%\end{array}$ & $55.7 \pm 14.9$ & $4.3 \pm 0.1$ \\
\hline \multicolumn{7}{|c|}{ The first generation from the Zard variety } \\
\hline 2 & Lotos & $23 / 03 \pm 5$ & $28 / 06 \pm 5$ & $\begin{array}{l}\text { cream-yel., } \\
\text { pink bl. up to } \\
50 \%\end{array}$ & $34.6 \pm 9$ & $4.3 \pm 0.2$ \\
\hline 3 & Krasnyj Krym & $23 / 03 \pm 5$ & $02 / 07 \pm 7$ & $\begin{array}{l}\text { yel., red. bl. } \\
\text { up to } 50 \%\end{array}$ & $67.0 \pm 10.6$ & $4.2 \pm 0.2$ \\
\hline 4 & Shalard 2 & $24 / 03 \pm 5$ & $02 / 07 \pm 5$ & $\begin{array}{l}\text { yel.-cream, } \\
\text { car. bl. up to } \\
10 \%\end{array}$ & $42.0 \pm 14.2$ & $4.4 \pm 0.3$ \\
\hline 5 & Arzami sladkij & $26 / 03 \pm 6$ & $05 / 07 \pm 12$ & $\begin{array}{l}\text { or., car. bl., } \\
\text { up to } 10 \%\end{array}$ & $32.0 \pm 7.4$ & $4.4 \pm 0.2$ \\
\hline 6 & $87-2$ & $26 / 03 \pm 3$ & $29 / 06 \pm 6$ & $\begin{array}{l}\text { cream.-yel., } \\
\text { pink bl. up to } \\
25 \%\end{array}$ & $33.8 \pm 5.7$ & $4.2 \pm 0.1$ \\
\hline 7 & Zapozdalyj & $01 / 04 \pm 4$ & $19 / 07 \pm 7$ & $\begin{array}{l}\text { yel., red. bl. } \\
\text { up to } 50 \%\end{array}$ & $36.4 \pm 8.2$ & $4.3 \pm 0.2$ \\
\hline \multicolumn{7}{|c|}{ The second generation from Zard variety } \\
\hline 8 & Boyarin & $29 / 03 \pm 4$ & $11 / 07 \pm 8$ & $\begin{array}{l}\text { or., ras. bl. } \\
\text { up to } 50 \%\end{array}$ & $41.8 \pm 4.7$ & $4.7 \pm 0.1$ \\
\hline 9 & Krokus & $19 / 03 \pm 8$ & $07 / 07 \pm 10$ & $\begin{array}{l}\text { br. - or., red. } \\
\text { bl. up to } 50 \%\end{array}$ & $73.2 \pm 15.7$ & $4.2 \pm 0.2$ \\
\hline 10 & $\begin{array}{l}\text { Pozdnocvetushchij } \\
1\end{array}$ & $29 / 03 \pm 9$ & $07 / 07 \pm 0$ & $\begin{array}{l}\text { cream-wh., } \\
\text { car. bl. up to } \\
25 \%\end{array}$ & $25.2 \pm 1.1$ & $4.0 \pm 0$ \\
\hline 11 & Yaltinec & $27 / 03 \pm 4$ & $28 / 06 \pm 8$ & $\begin{array}{l}\text { cream - yel., } \\
\text { red. bl. up to } \\
50 \%\end{array}$ & $60.0 \pm 12.7$ & $4.6 \pm 0.1$ \\
\hline 12 & $84-756$ & $24 / 03 \pm 6$ & $03 / 07 \pm 7$ & $\begin{array}{l}\text { yel.- or., red. } \\
\text { bl. up to } 25 \%\end{array}$ & $34.1 \pm 11$ & $4.1 \pm 0.1$ \\
\hline 13 & $84-315$ & $26 / 03 \pm 7$ & $11 / 07 \pm 7$ & $\begin{array}{l}\text { yel., red bl. } \\
\text { up to } 10 \%\end{array}$ & $33.9 \pm 8.1$ & $4.1 \pm 0.1$ \\
\hline 14 & $84-919$ & $31 / 03 \pm 7$ & $30 / 06 \pm 7$ & $\begin{array}{l}\text { or., red bl. up } \\
\text { to } 10 \%\end{array}$ & $44.6 \pm 9$ & $4.1 \pm 0.1$ \\
\hline 15 & $84-314$ & $21 / 03 \pm 7$ & $10 / 07 \pm 8$ & yel. & $37.9 \pm 5$ & $4.1 \pm 0.1$ \\
\hline 16 & $84-880$ & $26 / 03 \pm 6$ & $04 / 07 \pm 4$ & $\begin{array}{l}\text { or., car. bl. } \\
\text { up to } 50 \%\end{array}$ & $23.8 \pm 3.2$ & $4.4 \pm 0.2$ \\
\hline 17 & $84-643$ & $26 / 03 \pm 5$ & $03 / 07 \pm 8$ & $\begin{array}{l}\text { or., red bl. up } \\
\text { to } 25 \%\end{array}$ & $38.3 \pm 10.3$ & $4.1 \pm 0.1$ \\
\hline 18 & $99-414$ & $25 / 03 \pm 5$ & $06 / 07 \pm 5$ & yel. & $50.3 \pm 12.9$ & $4.4 \pm 0.1$ \\
\hline
\end{tabular}




\begin{tabular}{|c|c|c|c|c|c|c|}
\hline 19 & $84-383$ & $01 / 04 \pm 3$ & $09 / 07 \pm 6$ & yel. & $33.0 \pm 4.2$ & $4.1 \pm 0.1$ \\
\hline 20 & $84-953$ & $25 / 03 \pm 4$ & $30 / 06 \pm 7$ & $\begin{array}{l}\text { yel.- or., car. } \\
\text { bl. up to } 25 \%\end{array}$ & $52.0 \pm 5.1$ & $4.6 \pm 0.1$ \\
\hline 21 & $84-818$ & $22 / 03 \pm 5$ & $30 / 06 \pm 5$ & $\begin{array}{l}\text { yel.- or., ras. } \\
\text { bl. up to } 50 \%\end{array}$ & $50.4 \pm 21.6$ & $4.5 \pm 0.2$ \\
\hline 22 & $84-651$ & $22 / 03 \pm 5$ & $04 / 07 \pm 7$ & $\begin{array}{l}\text { yel.- or., red } \\
\text { bl. up to } 50 \%\end{array}$ & $59.2 \pm 6.4$ & $4.4 \pm 0.2$ \\
\hline 23 & $84-915$ & $08 / 04 \pm 6$ & - & - & - & - \\
\hline 24 & $84-679$ & $26 / 03 \pm 5$ & $10 / 07 \pm 9$ & $\begin{array}{l}\text { cream, pink } \\
\text { bl. up to } 10 \%\end{array}$ & $40.9 \pm 8$ & $4.0 \pm 0$ \\
\hline 25 & $84-783$ & $24 / 03 \pm 5$ & $28 / 06 \pm 6$ & yel. & $36.2 \pm 11.7$ & $4.3 \pm 0.2$ \\
\hline 26 & $84-949$ & $19 / 03 \pm 6$ & $26 / 06 \pm 7$ & $\begin{array}{l}\text { or., red bl. up } \\
\text { to } 25 \%\end{array}$ & $46.1 \pm 15.5$ & $4.2 \pm 0.1$ \\
\hline 27 & $84-768$ & $25 / 03 \pm 6$ & $01 / 07 \pm 5$ & yel.- or., & $48.8 \pm 2.8$ & $4.0 \pm 0$ \\
\hline 28 & $84-890$ & $25 / 03 \pm 4$ & $08 / 07 \pm 10$ & $\begin{array}{l}\text { yel.- or., red } \\
\text { bl. up to } 50 \%\end{array}$ & $35.5 \pm 7$ & $4.3 \pm 0.2$ \\
\hline 29 & $84-942$ & $23 / 03 \pm 5$ & $08 / 07 \pm 9$ & $\begin{array}{l}\text { yel. - or., red } \\
\text { bl. up to } 50 \%\end{array}$ & $54.4 \pm 18.4$ & $4.1 \pm 0.1$ \\
\hline 30 & $84-895$ & $29 / 03 \pm 6$ & $12 / 07 \pm 4$ & $\begin{array}{l}\text { yel.- or., } \\
\text { pink bl. } 10 \%\end{array}$ & $31.4 \pm 5.4$ & $4.0 \pm 0.1$ \\
\hline 31 & $84-941$ & $26 / 03 \pm 5$ & $03 / 07 \pm 6$ & $\begin{array}{l}\text { or., red bl. up } \\
\text { to } 50 \%\end{array}$ & $52.0 \pm 16.5$ & $4.2 \pm 0.2$ \\
\hline 32 & $84-951$ & $27 / 03 \pm 5$ & $27 / 06 \pm 6$ & $\begin{array}{l}\text { cream, car. } \\
\text { bl. } 25 \%\end{array}$ & $61.1 \pm 13.5$ & $4.6 \pm 0.2$ \\
\hline 33 & $84-952$ & $25 / 03 \pm 6$ & $05 / 07 \pm 3$ & $\begin{array}{l}\text { yel.- or., car. } \\
\text { bl. } 50-75 \%\end{array}$ & $54.4 \pm 18.9$ & $4.5 \pm 0$ \\
\hline 34 & $84-676$ & $26 / 03 / \pm 5$ & $30 / 06 \pm 16$ & - & - & - \\
\hline 35 & $84-875$ & $30 / 03 \pm 4$ & $01 / 07 \pm 7$ & $\begin{array}{l}\text { br.- or., red } \\
\text { bl., } 25-50 \%\end{array}$ & $22.6 \pm 5.2$ & $4.0 \pm 0$ \\
\hline 36 & $84-909$ & $28 / 03 \pm 4$ & $02 / 07 \pm 6$ & yel.- or., & $33.4 \pm 6.5$ & $4.4 \pm 0.2$ \\
\hline 37 & $84-986$ & $26 / 03 \pm 5$ & $03 / 07 \pm 6$ & $\begin{array}{l}\text { green- yel., } \\
\text { red bl. up to } \\
10 \%\end{array}$ & $34.5 \pm 8.7$ & $4.1 \pm 0.1$ \\
\hline 38 & $84-859$ & $25 / 03 \pm 5$ & $16 / 07 \pm 6$ & $\begin{array}{l}\text { yel.- or., car. } \\
\text { bl. } 25 \%\end{array}$ & $42.9 \pm 13.5$ & $4.0 \pm 0$ \\
\hline 39 & $84-624$ & $24 / 03 \pm 6$ & $11 / 07 \pm 9$ & $\begin{array}{l}\text { or., pink bl. } \\
\text { up to } 10 \%\end{array}$ & $50.0 \pm 10$ & $4.1 \pm 0.2$ \\
\hline 40 & $84-988$ & $26 / 03 \pm 4$ & $29 / 06 \pm 5$ & $\begin{array}{l}\text { yel., car. bl. } \\
5-10 \%\end{array}$ & $42.8 \pm 10.2$ & $4.3 \pm 0.3$ \\
\hline 41 & $84-784$ & $23 / 03 \pm 9$ & $06 / 07 \pm 3$ & $\begin{array}{l}\text { cream- yel., } \\
\text { pink bl. } 25 \%\end{array}$ & $35.8 \pm 2.6$ & $4.2 \pm 0.2$ \\
\hline 42 & $84-803$ & $28 / 03 \pm 5$ & $11 / 07 \pm 8$ & $\begin{array}{l}\text { cream- yel., } \\
\text { pink bl. } 10 \%\end{array}$ & $33.4 \pm 3.9$ & $4.4 \pm 0.1$ \\
\hline 43 & $84-769$ & $28 / 03 \pm 5$ & $11 / 07 \pm 8$ & $\begin{array}{l}\text { cream- yel., } \\
\text { red bl. up to } \\
10 \%\end{array}$ & $39.1 \pm 2.5$ & $4.4 \pm 0.2$ \\
\hline 44 & $84-922$ & $25 / 03 \pm 5$ & $10 / 07 \pm 7$ & $\begin{array}{l}\text { or., car. bl. } \\
5-10 \%\end{array}$ & $35.9 \pm 8$ & $4.4 \pm 0.2$ \\
\hline 45 & $84-684$ & $24 / 03 \pm 5$ & $01 / 07 \pm 11$ & $\begin{array}{l}\text { yel., red bl. } \\
\text { up to } 10 \%\end{array}$ & $59.2 \pm 0.4$ & $4.2 \pm 0.1$ \\
\hline
\end{tabular}




\begin{tabular}{|c|c|c|c|c|c|c|}
\hline 46 & $84-929$ & $26 / 03 \pm 6$ & $01 / 07 \pm 6$ & $\begin{array}{l}\text { or., red bl. up } \\
\text { to } 10 \%\end{array}$ & $20.0 \pm 5$ & $4.1 \pm 0.1$ \\
\hline 47 & $84-359$ & $25 / 03 \pm 8$ & $28 / 06 \pm 7$ & $\begin{array}{l}\text { cream- yel., } \\
\text { car. bl. } 10 \%\end{array}$ & $41.9 \pm 12$ & $4.3 \pm 0.4$ \\
\hline 48 & $84-639$ & $19 / 03 \pm 8$ & $12 / 07 \pm 8$ & $\begin{array}{l}\text { or., pink bl. } \\
\text { up to } 25 \%\end{array}$ & $44.2 \pm 11$ & $4.1 \pm 0.3$ \\
\hline 49 & $89-526$ & $27 / 03 \pm 6$ & $01 / 07 \pm 0$ & yel. & $40.4 \pm 7.6$ & $4.4 \pm 0.1$ \\
\hline 50 & $89-653$ & $29 / 03 \pm 7$ & $06 / 07 \pm 9$ & yel. & $53.3 \pm 12.5$ & $4.1 \pm 0.1$ \\
\hline 51 & $89-359$ & $26 / 03 \pm 5$ & $28 / 06 \pm 8$ & $\begin{array}{l}\text { cream- yel., } \\
\text { pink bl. } 25 \%\end{array}$ & $47.7 \pm 21.1$ & $4.2 \pm 0.1$ \\
\hline 52 & $89-363$ & $29 / 03 \pm 3$ & - & - & - & - \\
\hline 53 & $89-545$ & $26 / 03 \pm 4$ & $03 / 07 \pm 7$ & $\begin{array}{l}\text { yel.- or., red } \\
\text { bl. up to } 25 \%\end{array}$ & $47.5 \pm 13.7$ & $4.2 \pm 0.1$ \\
\hline 54 & $99-396$ & $01 / 04 \pm 5$ & $05 / 07 \pm 7$ & $\begin{array}{l}\text { yel.- or., car. } \\
\text { bl. } 25 \%\end{array}$ & $51.5 \pm 29.8$ & $4.1 \pm 0.2$ \\
\hline 55 & 8316 & $29 / 03 \pm 5$ & $28 / 06 \pm 6$ & $\begin{array}{l}\text { cream - yel., } \\
\text { red bl. } 25- \\
50 \%\end{array}$ & $56.2 \pm 8.4$ & $4.2 \pm 0.1$ \\
\hline 56 & 8319 & $31 / 03 \pm 6$ & $26 / 06 \pm 8$ & $\begin{array}{l}\text { or., car. bl. } \\
5-10 \%\end{array}$ & $28.3 \pm 0$ & $4.3 \pm 0$ \\
\hline 57 & 8457 & $29 / 03 \pm 6$ & $02 / 07 \pm 6$ & $\begin{array}{l}\text { cream- yel., } \\
\text { red bl. } 25- \\
50 \%\end{array}$ & $32.3 \pm 7.7$ & $4.4 \pm 0.2$ \\
\hline 58 & 8886 & $25 / 03 \pm 5$ & $10 / 07 \pm 11$ & $\begin{array}{l}\text { cream, red } \\
\text { bl. up to } 25 \%\end{array}$ & $49.3 \pm 10$ & $4.2 \pm 0.2$ \\
\hline 59 & 9678 & $28 / 03 \pm 3$ & - & - & - & - \\
\hline 60 & 10841 & $28 / 03 \pm 6$ & $06 / 07 \pm 5$ & $\begin{array}{l}\text { yel., red bl. } \\
\text { up to } 10 \%\end{array}$ & $44.1 \pm 9.9$ & $4.4 \pm 0.1$ \\
\hline 61 & 9-IV-1/15 & $22 / 03 \pm 7$ & $02 / 07 \pm 9$ & $\begin{array}{l}\text { or., red bl. } \\
25-50 \%\end{array}$ & $33.0 \pm 8.8$ & $4.1 \pm 0.1$ \\
\hline 62 & $99-354$ & $21 / 03 \pm 5$ & $30 / 06 \pm 1$ & $\begin{array}{l}\text { yel.- or., red } \\
\text { b1. } 25 \%\end{array}$ & $57.2 \pm 0.4$ & $4.5 \pm 0.1$ \\
\hline \multicolumn{7}{|c|}{ The third generation from Zard variety } \\
\hline 63 & $89-164$ & $20 / 03 \pm 7$ & $30 / 06 \pm 1$ & $\begin{array}{l}\text { yel.- or., red } \\
\text { bl. up to } 10 \%\end{array}$ & $58.9 \pm 16.5$ & $4.3 \pm 0.3$ \\
\hline 64 & $89-166$ & $20 / 03 \pm 7$ & $15 / 06 \pm 5$ & or. & $76.1 \pm 24.3$ & $4.5 \pm 0$ \\
\hline 65 & $89-169$ & $22 / 03 \pm 5$ & $14 / 07 \pm 3$ & yel.- or. & $60.3 \pm 0.1$ & $4.0 \pm 0.1$ \\
\hline
\end{tabular}

Note: the color of the basic skin - yel.-or. -Yellow-orange; yel.-cream. -Yellow cream; or.- orange; yel. - yellow; cream yel- cream yellow; br. or. - bright orange; cream - wh - creamy white; cream. - cream; green - yel. - greenish yellow;

the color of integumentary skin: red bl. - red blush; car. bl. - carmine blush; pink bl. - pink blush; ras. bl. - raspberryred blush;

The brightest cover color, covering up to $50 \%$ or more of the fruit surface, was observed in three varieties of the first generation (Krasnyj Krym, Zapozdalyj and Lotos). Among the genotypes of the second generation, $25.5 \%$ of varieties and hybrid forms are identified. Of these, orange or yellow-orange skin with an elegant blush covering half of the surface of the fruit, had varieties Boyarin, Krokus and hybrid forms 84-880,84-818,84-651,84-890, 84-942, 84-941, 84-952, 84-875. In the third generation, the plants were distinguished by orange fruits without a cover color or with a slight blush, covering up to $10 \%$ of the fruit surface. 
The best taste (with a tasting score of 4.5-4.7 points, on a 5-point scale) stands out two varieties (Boyarin and Krokus) and four hybrid forms (84-953, 84-818, 84-952, 99-354).

Apricot plants are also highly susceptible to diseases that cause declines and ultimately crop loss and tree death. The application of protective measures to preserve the harvest entails a deterioration in the environment in horticultural areas. Therefore, when creating more adaptive apricot varieties, it is important to select genotypes that are most resistant to pathogens harmful to plants (Moniliacinerea Bon. и Clasterosporium carpophilum (Lev.) Aderh.).

The analysis of the studied forms made it possible to identify the hybrid forms that are least susceptible to harmful fungal diseases (Table 2)

Table 3. Adaptive characteristics of varieties and breeding hybrids of apricot (2015-2019)

\begin{tabular}{|c|c|c|c|c|c|}
\hline № & $\begin{array}{l}\text { Variety, hybrid } \\
\text { form }\end{array}$ & $\begin{array}{l}\text { Productivity, } \\
\text { score }\end{array}$ & $\begin{array}{c}\text { Monilia } \\
\text { affection, } \\
\text { score }\end{array}$ & $\begin{array}{l}\text { Clotterosporium } \\
\text { affection, score }\end{array}$ & $\begin{array}{c}\text { Drought } \\
\text { resistance, } \\
\text { score }\end{array}$ \\
\hline 1 & 2 & 3 & 4 & 5 & 6 \\
\hline 1 & Krymskij Amur (k) & $2.8 \pm 0.6$ & $2.3 \pm 1.1$ & $1.6 \pm 0.6$ & $3.3 \pm 0.3$ \\
\hline \multicolumn{6}{|c|}{ The first generation from the Zard variety } \\
\hline 2 & Shalard 2 & $2.8 \pm 0.6$ & $2.3 \pm 1.1$ & $2.0 \pm 0.8$ & $3.1 \pm 0.4$ \\
\hline 3 & Arzami sladkij & $1.3 \pm 0.7$ & $1.5 \pm 1.0$ & $2.0 \pm 0.4$ & $3.4 \pm 0.5$ \\
\hline 4 & Zapozdalyj & $1.6 \pm 0.5$ & $2.0 \pm 1.2$ & $1.6 \pm 0.7$ & $3.4 \pm 0.3$ \\
\hline 5 & Krasnyj Krym & $2.3 \pm 0.6$ & $2.8 \pm 1.1$ & $1.3 \pm 1.0$ & $3.7 \pm 0.5$ \\
\hline 6 & Lotos & $2.4 \pm 0.8$ & $3.0 \pm 1.9$ & $2.2 \pm 0.8$ & $3.5 \pm 0.4$ \\
\hline 7 & $87-2$ & $2.8 \pm 0.5$ & $1.8 \pm 1.0$ & $2.2 \pm 0.7$ & $3.6 \pm 0.3$ \\
\hline \multicolumn{6}{|c|}{ The second generation from Zard variety } \\
\hline 8 & Boyarin & $2.3 \pm 0.5$ & $1.6 \pm 0.9$ & $1.2 \pm 0.5$ & $2.9 \pm 0.2$ \\
\hline 9 & Krokus & $2.6 \pm 1.4$ & $2.4 \pm 1.7$ & $2.8 \pm 0.8$ & $3.7 \pm 0.2$ \\
\hline 10 & $\begin{array}{l}\text { Pozdnocvetushchij } \\
1\end{array}$ & $0.7 \pm 0.2$ & $2.0 \pm 1.5$ & $1.5 \pm 0.8$ & $3.5 \pm 0.4$ \\
\hline 11 & Yaltinec & $2.9 \pm 0.4$ & $2.0 \pm 0.9$ & $1.3 \pm 0.8$ & $3.8 \pm 0.7$ \\
\hline 12 & $84-756$ & $2.2 \pm 0.6$ & $2.6 \pm 1.8$ & $1.6 \pm 0.9$ & $3.5 \pm 0.6$ \\
\hline 13 & $84-315$ & $2.0 \pm 0.7$ & $3.1 \pm 1.3$ & $1.8 \pm 0.8$ & $3.7 \pm 0.3$ \\
\hline 14 & $84-919$ & $0.8 \pm 0.4$ & $1.1 \pm 1.0$ & $1.2 \pm 0.6$ & $3.5 \pm 0.5$ \\
\hline 15 & $84-314$ & $2.1 \pm 0.5$ & $3.5 \pm 1.2$ & $1.8 \pm 0.5$ & $3.2 \pm 0.3$ \\
\hline 16 & $84-880$ & $1.4 \pm 0.6$ & $1.0 \pm 0.8$ & $1.5 \pm 0.5$ & $3.2 \pm 0.3$ \\
\hline 17 & $84-643$ & $2.1 \pm 0.6$ & $1.5 \pm 1.4$ & $1.6 \pm 0.4$ & $3.8 \pm 0.5$ \\
\hline 18 & $99-414$ & $2.5 \pm 0.6$ & $2.8 \pm 1.6$ & $1.4 \pm 0.5$ & $3.6 \pm 0.4$ \\
\hline 19 & $84-383$ & $2.4 \pm 1.0$ & $0.3 \pm 0.2$ & $1.1 \pm 0.5$ & $3.4 \pm 0.7$ \\
\hline 20 & $84-953$ & $1.5 \pm 0.3$ & $2.2 \pm 1.0$ & $0.9 \pm 0.4$ & $2.9 \pm 0.4$ \\
\hline 21 & $84-818$ & $2.3 \pm 0.6$ & $2.4 \pm 1.4$ & $1.4 \pm 0.9$ & $3.9 \pm 0.4$ \\
\hline 22 & $84-651$ & $3.3 \pm 0.3$ & $2.2 \pm 1.5$ & $1.8 \pm 0.6$ & $3.6 \pm 0.1$ \\
\hline 23 & $84-915$ & $1.0 \pm 0$ & $0.5 \pm 0.3$ & $1.3 \pm 0.6$ & $3.3 \pm 0.4$ \\
\hline 24 & $84-679$ & $2.5 \pm 0.8$ & $2.7 \pm 1.1$ & $2.2 \pm 0.6$ & $3.3 \pm 0.3$ \\
\hline 25 & $84-783$ & $2.6 \pm 0.6$ & $2.5 \pm 2.2$ & $1.5 \pm 0.5$ & $3.4 \pm 0.4$ \\
\hline 26 & $84-949$ & $0.8 \pm 0.4$ & $2.8 \pm 1.4$ & $1.9 \pm 0.9$ & $3.4 \pm 0.5$ \\
\hline 27 & $84-768$ & $1.7 \pm 0.7$ & $1.9 \pm 1.2$ & $1.4 \pm 0.6$ & $3.6 \pm 0.2$ \\
\hline 28 & $84-890$ & $2.2 \pm 0.5$ & $3.1 \pm 1.2$ & $1.5 \pm 0.6$ & $3.4 \pm 0.4$ \\
\hline 29 & $84-942$ & $2.6 \pm 0.8$ & $2.2 \pm 1.5$ & $1.4 \pm 0.8$ & $3.7 \pm 0.6$ \\
\hline 30 & $84-895$ & $2.0 \pm 0$ & $2.3 \pm 1.0$ & $1.5 \pm 0.6$ & $3.5 \pm 0.4$ \\
\hline 31 & $84-941$ & $2.3 \pm 0.6$ & $3.2 \pm 1.0$ & $1.8 \pm 0.8$ & $3.4 \pm 0.4$ \\
\hline
\end{tabular}




\begin{tabular}{|c|c|c|c|c|c|}
\hline 32 & $84-951$ & $2.2 \pm 0.5$ & $1.9 \pm 1.1$ & $1.5 \pm 0.5$ & $3.5 \pm 0.3$ \\
\hline 33 & $84-952$ & $2.8 \pm 1.3$ & $2.4 \pm 1.0$ & $1.2 \pm 0.6$ & $3.4 \pm 0.5$ \\
\hline 34 & $84-676$ & $1.8 \pm 0.5$ & $1.0 \pm 0.8$ & $1.7 \pm 0.6$ & $3.2 \pm 0.5$ \\
\hline 35 & $84-875$ & $1.4 \pm 0.8$ & $1.2 \pm 1.0$ & $1.3 \pm 0.6$ & $3.1 \pm 0.6$ \\
\hline 36 & $84-909$ & $1.1 \pm 0.3$ & $0.5 \pm 0.4$ & $1.3 \pm 0.4$ & $3.2 \pm 0.3$ \\
\hline 37 & $84-986$ & $2.4 \pm 0.6$ & $2.0 \pm 1.6$ & $1.4 \pm 0.7$ & $3.4 \pm 0.4$ \\
\hline 38 & $84-859$ & $2.9 \pm 0.5$ & $2.0 \pm 1.0$ & $1.4 \pm 0.5$ & $3.2 \pm 0.4$ \\
\hline 39 & $84-624$ & $2.2 \pm 0.7$ & $1.4 \pm 0.9$ & $1.7 \pm 1.0$ & $3.7 \pm 0.3$ \\
\hline 40 & $84-988$ & $3.8 \pm 0.5$ & $2.6 \pm 0.9$ & $2.5 \pm 1.1$ & $3.6 \pm 0.7$ \\
\hline 41 & $84-784$ & $2.0 \pm 0.4$ & $2.0 \pm 1.4$ & $1.3 \pm 0.6$ & $3.7 \pm 0.6$ \\
\hline 42 & $84-803$ & $2.1 \pm 1.0$ & $1.4 \pm 1.2$ & $1.5 \pm 0.4$ & $3.6 \pm 0.2$ \\
\hline 43 & $84-769$ & $1.7 \pm 0.8$ & $1.5 \pm 1.2$ & $1.0 \pm 0.8$ & $3.9 \pm 0.5$ \\
\hline 44 & $84-922$ & $2.2 \pm 1.1$ & $2.6 \pm 1.6$ & $1.2 \pm 0.8$ & $3.4 \pm 0.5$ \\
\hline 45 & $84-684$ & $1.0 \pm 0.3$ & $2.0 \pm 1.0$ & $1.8 \pm 0.3$ & $3.4 \pm 0.3$ \\
\hline 46 & $84-929$ & $0.2 \pm 0.1$ & $1.7 \pm 0.8$ & $0.9 \pm 0.4$ & $3.2 \pm 0.8$ \\
\hline 47 & $84-359$ & $1.6 \pm 0.6$ & $3.2 \pm 1.8$ & $2.1 \pm 1.2$ & $3.4 \pm 0.5$ \\
\hline 48 & $84-639$ & $3.7 \pm 0.9$ & $3.7 \pm 1.3$ & $1.8 \pm 0.3$ & $3.7 \pm 0.4$ \\
\hline 49 & $89-526$ & $0.7 \pm 0.2$ & $1.0 \pm 0.5$ & $1.6 \pm 0.8$ & $3.9 \pm 0.2$ \\
\hline 50 & $84-803$ & $0.9 \pm 0.6$ & $1.0 \pm 0.8$ & $1.5 \pm 0.7$ & $3.8 \pm 0.3$ \\
\hline 51 & 89-359 & $2.6 \pm 0.6$ & $2.8 \pm 1.3$ & $1.4 \pm 0.7$ & $3.7 \pm 0.3$ \\
\hline 52 & $89-363$ & - & $0.5 \pm 0.4$ & $1.1 \pm 0.9$ & $3.5 \pm 0.4$ \\
\hline 53 & $89-545$ & $1.3 \pm 0.8$ & $0.8 \pm 0.3$ & $1.0 \pm 0.9$ & $3.2 \pm 0.3$ \\
\hline 54 & $99-396$ & $3.1 \pm 0.6$ & $1.9 \pm 1.0$ & $1.8 \pm 0.7$ & $3.6 \pm 0.2$ \\
\hline 55 & 8316 & $2.8 \pm 0.6$ & $2.2 \pm 1.5$ & $1.5 \pm 0.6$ & $3.5 \pm 0.4$ \\
\hline 56 & 8319 & $2.2 \pm 1.4$ & $1.7 \pm 1.0$ & $1.9 \pm 0.5$ & $3.5 \pm 0.3$ \\
\hline 57 & 8457 & $2.2 \pm 0.9$ & $1.7 \pm 1.3$ & $1.1 \pm 0.4$ & $3.5 \pm 0$ \\
\hline 58 & 8886 & $1.2 \pm 0.4$ & $3.0 \pm 0.7$ & $1.2 \pm 0.7$ & $3.5 \pm 0.7$ \\
\hline 59 & 9678 & - & $2.0 \pm 1.9$ & $1.2 \pm 1.0$ & $3.2 \pm 0.5$ \\
\hline 60 & 10841 & $2.2 \pm 0.4$ & $0.7 \pm 0.6$ & $1.3 \pm 0.4$ & $3.4 \pm 0.7$ \\
\hline 61 & $9-I V-1 / 15$ & $3.2 \pm 0.7$ & $3.4 \pm 1.6$ & $1.6 \pm 0.8$ & $3.5 \pm 0.4$ \\
\hline 62 & $99-354$ & $2.1 \pm 1.1$ & $3.0 \pm 0.7$ & $2.1 \pm 0.7$ & $3.3 \pm 0.4$ \\
\hline \multicolumn{6}{|c|}{ The third generation from Zard variety } \\
\hline 63 & $89-164$ & $0.9 \pm 0.2$ & $3.3 \pm 1.0$ & $2.0 \pm 0.4$ & $3.5 \pm 0.8$ \\
\hline 64 & $89-166$ & $1.8 \pm 0.3$ & $2.5 \pm 1.6$ & $2.2 \pm 0.7$ & $3.8 \pm 0.3$ \\
\hline 65 & $89-169$ & $2.8 \pm 0.9$ & $1.4 \pm 0.9$ & $1.6 \pm 0.4$ & $3.7 \pm 0.2$ \\
\hline
\end{tabular}

The least susceptible to moniliosis and klyasterosporiosis are 20 genotypes, moreover, in the first generation one variety is Zapozdalyj, in the third one hybrid form is $89-169$, and in the second - 18 (Boyarin, 84-919, 84-880, 84-643, 84-383, 84-915, 84-768, 84- 952, 84-875, $84-909$, etc.). Field resistance to moniliosis is distinguished by $60.0 \%$ of hybrid forms, to clasterosporiosis - $64.6 \%$. The study of drought resistance of the created hybrid forms is an important direction due to conditions of increasing dry growing seasons. Among the studied genotypes of drought resistance, 21 hybrid forms are distinguished.

Fruits play an important role in human nutrition and are an essential part of their diet. Therefore, one of the main directions for the development of the fruit-growing industry, not only in our country, but also in other states, is to provide the population with high-quality fruit and berry products.

The nutritional and medicinal value of the fruits lies in their rich chemical composition $[16,17]$. As a result of studying the chemical composition of the fruits of seven promising hybrid forms, according to the complex of biochemical indicators (dry substances, ascorbic 
acid, flavonols, leukoanthocyanins, the sum of phenolic compounds, etc.), the Boyarin variety and four hybrid forms (84-951, 84-818, 10841 and 8316) were distinguished. The highest content of dry matter (20.25-23.1\%) was found in fruits of the Boyarin variety and hybrid forms 10841, 8316; ascorbic acid (10.74-15.84 mg / $100 \mathrm{~g}$ ) - in hybrid forms 84951.84-818 and 8316.; flavonols (14.95-16.6 mg / $100 \mathrm{~g}$ ) - in hybrid forms 84-951 and 10841.

\section{Conclusion}

Due to conducted studies was revealed that the main directions of apricot breeding at the modern level are complex studies of genetic resources to determine the possibility of the most effective targeted selection of parental pairs during creating varieties with high commercial qualities of fruits, high-yielding, resistant to abiotic and biotic stress factors, adapted to intensive cultivation technologies.

It has been determined that the Zard variety, up to the second generation, transmits to its offspring late flowering and small fruiting.

The most promising apricot genotypes for cultivation in areas with unstable weather conditions in Southern Russia have been identified: Boyarin, Yaltinec, 84-951, 89-653, 99$396,8316,10841,84-941$, they are characterized by large or medium-sized fruits, good taste (tasting score 4.1-4.7 points) and attractive appearance, late or mid-late flowering of plants. According to a set of chemical indicators, the Boyarin variety and hybrid forms (84-951, 10841, 84-818 and 8316) are distinguished.

Variety Yaltinets and hybrid form 89-169 are distinguished by low susceptibility to fungal diseases (moniliosis and klyasterosporia) and high resistance to drought, which are not inferior in yield to the control variety Krymskiy Amur.

The following varieties and hybrid forms are of interest for breeding - for late flowering: Zapozdalyi, 8457, 84-383, 84-895, 8319, 84-875; weak susceptibility to fungal diseases (clasterosporium and moniliosis): 84-919, 84-880, 84-383, 84-915, 84-875, 84-909, 84-803, 84-769, 84-803, 89- 545, 10841; drought resistance: Crocus, Yaltinets, 87-2, 84-651. 84-942, 84-988, 84-639, 89-359, 89-526, 89-166 and 89-169 - against the background of dry growing seasons, their productivity was at the level of the control variety and higher (2.6-3.9 points).

\section{References}

1. A. Smykov, ActaHortic., 1139, 79-84 (2016) https://doi.org/10.17660/ActaHortic.2016. 1139.14

2. V. Gorina, V. Korzin, N. Mesyats. ActaHortic.,1255, 153-156 (2019)https://doi.org/ 10.17660/ActaHortic.2019.1255.24

3. D. Bassi, M. Rizzo, S. Foschi. Acta Hortic.,862, 151-158 (2010) https://doi.org/10.17660/ ActaHortic. 2010.862.23

4. T. Latsko, ActaHortic.,1139, 71-78 (2016) https://doi.org/10.17660/ActaHortic.2016. 1139.13

5. B. Gouble, P. Reling, C.M.G.C. Renard, J.M. Broquaire, C. Chamet, J.M. Audergon. ActaHortic., 1214, 53-58 (2018) https://doi.org/10.17660/ActaHortic.2018.1214.9

6. Y. Serena Park, Database 'FAOSTAT', (2019)http://www.fao.org/faostat/en/\#home

7. S.Bureau, B.Gouble, J.M.Audergon, S. Costa, S.Tozanli, ActaHortic.,1214, 39-44(2018) https://doi.org/10.17660/ActaHortic.2018.1214.7

8. B.Krska, ActaHortic.,1172,201-204 (2017) https://doi.org/10.17660/ActaHortic.2017. $\underline{1172.38}$

9. V.Gorina, A. Richter. ActaHortic.,1208,183-190. (2018) https://doi.org/10.17660 /ActaHortic.2018.1208.24 
10. V.Gorina, V.Korzin, N. Mesyats.ActaHortic.,1282,25-30 (2020) https://doi.org/10.17660/ ActaHortic.2020.1282.5

11. G. Fremondiere, A. Blanc, F. Gilles, G. Clauzel, J.M. Broquaire, G. Roch, B. Gouble, S. Bureau, C. Pitiot, J.M. Audergon. ActaHortic.,1214, 207-210(2018) https://doi.org/10. $\underline{17660 / \text { ActaHortic.2018.1214.36 }}$

12. V.Gorina, V. Korzin, ActaHortic., 1139, 363-368 (2016) http://doi.org/10.17660/ ActaHortic.2016.1139.63

13. L. Szalay, M. Ladányi, V. Hajnal, A. Pedryc, M. Tóth. Hort. Sci. (Prague), 43, 134-141. (2016)https://doi.org/10.17221/161/2015-HORTSCI

14. M. Szymajda, K. Pruski, E.Zurawicz, M. Sitarek,Can. J. Plant Sci.,93(2), 191-198 (2013) https://doi.org/10.4141/cjps2012-238

15. V.V. Korzin,Taurida Herald of the Agrarian Sciences, 3(15),65-71 (2018) http://doi.org/ 10.25637/TVAN.2018.03.07.

16. S. Ali, T. Masud, K.S.Abbasi, Sci. Hortic.,130(2), 386-392 (2011) https://doi.org/10.1016 j.scienta.2011.05.040

17. A. Leccese, S. Bartolini, R.Viti, Plant Foods Hum. Nutr., 67, 317-325 (2012) https://doi. $\underline{\text { org } / 10.1007 / \mathrm{s} 11130-012-0314-0}$ 